ARTICLE

Received 9 Nov 2012 | Accepted 24 May 2013 | Published 3 July $2013 \quad$ DOI: 10.1038/ncomms3057

\title{
Connection between Bell nonlocality and Bayesian game theory
}

Nicolas Brunner ${ }^{1,2} \&$ Noah Linden 3

In 1964, Bell discovered that quantum mechanics is a nonlocal theory. Three years later, in a seemingly unconnected development, Harsanyi introduced the concept of Bayesian games. Here we show that, in fact, there is a deep connection between Bell nonlocality and Bayesian games, and that the same concepts appear in both fields. This link offers interesting possibilities for Bayesian games, namely of allowing the players to receive advice in the form of nonlocal correlations, for instance using entangled quantum particles or more general nosignalling boxes. This will lead to novel joint strategies, impossible to achieve classically. We characterize games for which nonlocal resources offer a genuine advantage over classical ones. Moreover, some of these strategies represent equilibrium points, leading to the notion of quantum/no-signalling Nash equilibrium. Finally, we describe new types of question in the study of nonlocality, namely the consideration of nonlocal advantage given a set of Bell expressions.

\footnotetext{
${ }^{1}$ Département de Physique Théorique, Université de Genève, 1211 Genève, Switzerland. ${ }^{2}$ H.H. Wills Physics Laboratory, University of Bristol, Bristol BS8 $1 T L$, UK. ${ }^{3}$ School of Mathematics, University of Bristol, Bristol BS8 1TW, UK. Correspondence and requests for materials should be addressed to N.B. (email: nicolas.brunner@unige.ch).
} 
$\mathrm{n}$ several occasions in the history of science, different areas of research, sharing a priori nothing in common and sometimes belonging to completely different fields of science, were shown to be closely related. In certain cases, these links turned out to be spectacular and tremendously fruitful, such as the connection between differential geometry and relativity. In the present paper, we discuss such a link, albeit a much more modest one, between Bell nonlocality and the theory of Bayesian games-also referred to as games with incomplete information.

Nonlocality is arguably among the most dramatic and counterintuitive features of quantum mechanics. In a nutshell, quantum theory is at odds with the principle of locality, which states that an object is influenced directly only by its immediate surroundings, and not by remotely located objects. Two remote observers sharing a pair of entangled quantum particles can establish correlations that evade any possible explanation in classical physics. On the one hand, a signal is excluded, as it would have to travel faster than light. On the other hand, the correlated behaviour is not the result of a pre-established strategy, as demonstrated by Bell in 1964 (ref. 1). Notably, this phenomenon of quantum nonlocality, confirmed experimentally ${ }^{2}$ via the violation of so-called Bell inequalities, turns out to be useful in practice, in particular for information processing ${ }^{3-5}$. More recently a theory of generalized nonlocal correlations has been developed $^{6,7}$, which has direct impact on fundamental questions in the foundations of quantum mechanics ${ }^{8,9}$.

In a completely different area, but only 3 years after Bell's ground-breaking discovery, Harsanyi ${ }^{10}$ developed a framework for games with incomplete information, that is, games in which players have only partial information about the setting in which the game is played. For instance, each player may have some private information, such as his payoff, unknown to other players. In general, players also have the possibility of using a common piece of advice (originating, e.g., from an advisor), allowing for correlated strategies. Harsanyi's discovery marked the start of Bayesian game theory, which now has a prominent role in game theory and in economics, used in particular to model auctions.

Here we discuss a strong connection between Bayesian games and Bell nonlocality, highlighting the fact that similar concepts appear in both fields. First, the fact that in a Bayesian game players have private information (a type) that is unknown to the other players coincides precisely with the notion of locality in physics. Second, the fact that the common advice does not allow a player to obtain information about the private information of other players is precisely the concept of no-signalling in physics. In other words, the physical resources available to the advisor must be non-signalling. These include local (i.e., classical) resources, as well as nonlocal resources, such as entangled quantum particles. Importantly, players having access to nonlocal resources can outperform players having access to the most general classical resources. From a more formal viewpoint, we will see that the normal form of a Bayesian game can be reformulated as a Bell test scenario. This will provide a general characterization of games where nonlocal resources provide an advantage over classical ones. These include for instances cases where the payoff function of the players corresponds to a Bell inequality, as first discussed by Cheon and Iqbal $^{11}$, and further developed in refs 12-17. However, we will see that there exist much more general situations (in which none of the payoffs functions corresponds to a Bell inequality) where nonlocal resources, e.g., entanglement, provide nevertheless an advantage over any classical strategy. Notably, some of these nonlocal strategies represent equilibrium points, termed quantum Nash equilibria or non-signalling Nash equilibria. To illustrate these ideas, we discuss several simple examples. Finally, we emphasize that, for the class of games discussed here (i.e., Bayesian games), quantum mechanics provides a clear and indisputable advantage over classical resources, in the most general sense. This is in contrast with some previous approaches to quantum games ${ }^{18}$, based on non-Bayesian games (or complete games, such as Prisoner's dilemma), for which a quantum advantage is achieved only under specific restrictions, the relevance of which has been much debated $^{19-22}$.

\section{Results}

From Bayesian games to Bell inequalities. Let us start with the normal form representation of a game. At this stage, one needs to specify the number of players, the set of possible strategies for each player and the payoff function for each player. To model Bayesian games, Harsanyi proposed to introduce Nature as an additional player to the game. In particular, Nature assigns to each player a type, chosen from a given set. The type of each player is generally unknown to other players, and determines, for instance, his payoff function. The set of possible types for each player is thus also part of the definition of a Bayesian game. More formally, the normal form representation of a Bayesian game is given by the following ingredients $^{23}$ : (i) the number of players $N$, (ii) a set of states of nature $\Omega$, with a prior $\mu(\Omega)$, (iii) for each player $i$, a set of actions $\mathcal{A}_{i}$, (iv) for each player $i$, a set of types $\chi_{i}$, (v) for each player $i$, a mapping $\tau_{i}: \Omega \rightarrow \chi_{i}$ and (vi) for each player $i$, a payoff function $f_{i}: \Omega \times \mathcal{A}_{1} \times \ldots \times \mathcal{A}_{N} \rightarrow \mathbb{R}$, determining the score of the player for any possible combination of types and actions.

In the following, we shall focus on the case of two players, for simplicity. The variable $A_{1}=0,1, \ldots k_{1}$ denotes the possible actions of party 1 , and $X_{1}=0,1, \ldots m_{1}$ denotes the possible types of party 1 etc. We will also consider that the possible states of Nature are simply the combination of all possible types (for all players), that is $\Omega=\left(\chi_{1}, \chi_{2}\right)$. To play the game, each player should decide on a particular strategy to follow. A pure strategy then consists in associating an action for every possible type, i.e., a mapping $s_{i}: \chi_{i} \rightarrow \mathcal{A}_{i}$. More generally, players may use a probabilistic strategy, hence it is convenient to define a probability of an action given a type, i.e., $P\left(A_{i} \mid X_{i}\right)$. An important feature of the game is then the average payoff function, or the average score, for each player. For player $i$ this is given by

$$
F_{i}=\sum \mu\left(X_{1}, X_{2}\right) P\left(A_{1}, A_{2} \mid X_{1}, X_{2}\right) f_{i}\left(X_{1}, X_{2}, A_{1}, A_{2}\right)
$$

where the sum goes over all variables $X_{1}, X_{2}, A_{1}, A_{2}$. Note that if the players use pure or independent strategies, then $P\left(A_{1}, A_{2} \mid X_{1}\right.$, $\left.X_{2}\right)=P\left(A_{1} \mid X_{1}\right) P\left(A_{2} \mid X_{2}\right)$. However, in certain cases the players may adapt their strategy depending on a piece of advice. The latter is delivered to all players by an advisor. This opens the possibility for the players to adopt correlated strategies, which can outperform independent strategies. There are various forms that advice can take. For example in the case of correlated classical advice, the advice is represented by a classical variable, $\lambda$, with prior $\rho(\lambda)$. Each player can then choose a strategy depending on his type and on $\lambda$. In general for classical correlated strategies, we have that

$$
\begin{gathered}
P\left(A_{1}, A_{2} \mid X_{1}, X_{2}\right)=\sum_{\lambda} \rho(\lambda) P\left(A_{1} \mid X_{1}, \lambda\right) P\left(A_{2} \mid X_{2}, \lambda\right) \\
\neq P\left(A_{1} \mid X_{1}\right) P\left(A_{2} \mid X_{2}\right)
\end{gathered}
$$

An important point in what follows (and not just in the case of classical advice) is that the advice must be independent of the state of Nature, that is, the choice of types is unknown to the advisor. This enforces the following condition

$$
P\left(A_{1} \mid X_{1}, X_{2}\right)=\sum_{A_{2}} P\left(A_{1}, A_{2} \mid X_{1}, X_{2}\right)=P\left(A_{1} \mid X_{1}\right)
$$

which states that the marginal of player 1 does not depend on the type of player 2; a similar condition holds for the marginal of 
player 2. In the context of Bell nonlocality (as we shall see below), the above condition plays a prominent role. It is referred to as the 'no-signalling' condition, which imposes that the correlations $P\left(A_{1}, A_{2} \mid X_{1}, X_{2}\right)$ do not allow for player 2 to signal instantaneously to player 1 , and thus respect causality and are not in conflict with relativity. We note that in the context of games, the situation in which the advice can depend on the types has been considered $^{24}$; physically this situation is however not so relevant for us, as it involves signalling.

To analyse games it is often useful to define the set of all possible pairs of payoff functions $\left\{F_{1}, F_{2}\right\}$, considering all possible strategies. It is convenient to represent geometrically the space of possible payoff functions ${ }^{23}$, i.e., here the set of points in $\mathbb{R}^{2}$ with coordinates $\left(F_{1}, F_{2}\right)$. In case the players share classical advice (and the number of possible strategies is finite), this space is a convex polytope. The space can then be conveniently characterized with a finite set of linear inequalities of the form

$$
\sum_{j=1}^{N=2} \beta_{j} F_{j} \leq \beta_{0}
$$

where $\beta_{j}$ are real numbers. These inequalities define the facets of this polytope.

A notion of particular importance in game theory is that of a Nash equilibrium. In case the game features an advisor, there is a more refined notion of correlated equilibrium ${ }^{25}$. Players achieve a correlated equilibrium for a given set of strategies if each player has no incentive to change strategy, that is, his average payoff will not increase by choosing any other possible strategy (keeping the other player's strategy fixed).

We shall see now that the above scenario is closely related to that of a Bell test. For simplicity, we will focus on a Bell test with two parties, although more parties can be considered. We consider two separated observers, Alice and Bob, sharing a physical resource distributed by a central source (see Fig. 1). Each observer receives a question (chosen in a given set) to which she/ he is asked to give an answer. In more physical terms, these questions should be understood as measurement settings, and the corresponding answers as measurement outcomes. Importantly each observers knows only his own question, and does not know which question the other observers receive. To make the analogy with a Bayesian game, the questions here correspond to the type of each player, while the answers correspond to the actions. Hence we will denote by $X_{1}$ and $X_{2}$ the questions of Alice and Bob, respectively, and by $A_{1}$ and $A_{2}$ the corresponding answers. After repeating the above operation a large number of times, the

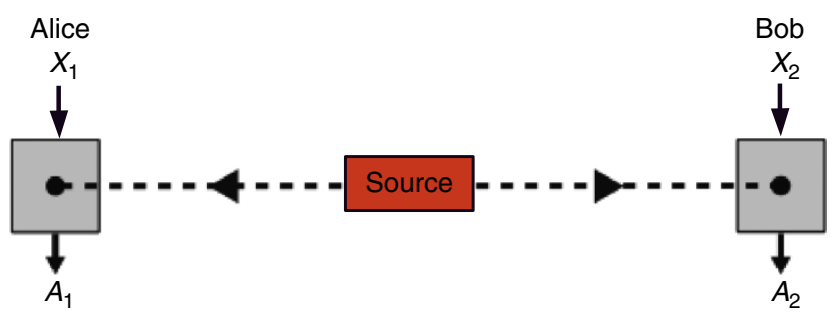

Figure 1 | Bell inequality test scenario. A source distributes physical resources to two distant players, Alice and Bob. Each player receives a question, denoted $X_{1}$ for Alice and $X_{2}$ for Bob, and should then provide an answer, denoted $A_{1}$ for Alice and $A_{2}$ for Bob. The experiment is characterized by a joint probability distribution $P\left(A_{1}, A_{2} \mid X_{1}, X_{2}\right)$. When the distribution $P\left(A_{1}, A_{2} \mid X_{1}, X_{2}\right)$ violates a Bell inequality, the observed correlations are nonlocal. statistics of the experiment can be computed, resulting in the joint probability distribution

$$
P\left(A_{1}, A_{2} \mid X_{1}, X_{2}\right)
$$

which represents the probability of observing a pair of answers $A_{1}, A_{2}$, given a pair of questions $X_{1}, X_{2}$.

In a Bell test, the goal is loosely speaking to capture the strength and the nature of the correlations observed in the experiment. In general, this may depend on the kind of physical resource distributed by the source to the observers. A case of particular importance is that of a classical source (i.e., a source of classical particles). In particular, the particles can be thought of as carrying information about a common strategy, which will eventually lead to a correlated behaviour in the experiment. The statistics of any experiment involving a classical source can be written as

$$
P\left(A_{1}, A_{2} \mid X_{1}, X_{2}\right)=\int \mathrm{d} \lambda \rho(\lambda) P\left(A_{1} \mid X_{1}, \lambda\right) P\left(A_{2} \mid X_{2}, \lambda\right)
$$

where the variable $\lambda$ (distributed according to the prior $\rho(\lambda)$, with $\int \mathrm{d} \lambda \rho(\lambda)=1$ ) represents the common strategy, that is, the information distributed from the source to all the observers. From the point of view of games, the variable $\lambda$ represents the advice. Thus, the source models the advisor. Importantly, all possible strategies for players receiving classical advice (i.e., from a classical advisor) are of this form.

In his 1964 ground-breaking work, Bell discovered that the correlations obtained in any experiment involving a classical source are constrained. More formally, any statistics of the form (6) satisfies a set of inequalities, now known as Bell inequalities ${ }^{1}$. Generally, a Bell inequality is based on a linear expression of the joint probabilities (5), of the form

$$
S=\sum_{X_{1}, X_{2}, A_{1}, A_{2}} \alpha_{X_{1}, X_{2}, A_{1}, A_{2}} P\left(A_{1}, A_{2} \mid X_{1}, X_{2}\right)
$$

where $\alpha_{X_{1}, X_{2}, A_{1}, A_{2}}$ are real numbers. The maximum of $S$ over all possible strategies of the form (6) is called the local bound of the inequality $L$. Putting all together, a Bell inequality then reads

$$
S \leq L
$$

Now we come to an important point. The average payoff function (for a given player $i$ ) is essentially a Bell expression. Indeed, equations (1) and (7) are exactly of the same form, with

$$
\mu\left(X_{1}, X_{2}\right) f_{i}\left(X_{1}, X_{2}, A_{1}, A_{2}\right)=\alpha_{X_{1}, X_{2}, A_{1}, A_{2}} .
$$

Hence to any average payoff function $F_{i}$ can be associated a Bell expression $S_{i}$. Moreover, in the presence of a classical advisor, the following condition must hold: $F_{i} \leq L$, where $L$ is the local bound of the Bell expression associated with $F_{i}$.

More generally, note that the above reasoning also applies to any linear combination of the payoff functions. In particular, the facets of the space of payoff functions, of the form (4), can also be associated with a Bell expression $S$. Thus, in the case of a classical advisor, the condition (4) can be seen as a Bell inequality, with Bell expression $S=\sum \beta_{j} F_{j}$ and local bound $L=\beta_{0}$.

To summarize, the payoff function of a Bayesian game is basically a Bell expression, and is hence limited by Bell's inequality for any strategy involving a classical advisor. More generally, this applies to linear combinations of payoff functions, such as those corresponding to the facets of the space of payoff functions. Next we shall move to quantum mechanics, for which the situation turns out to be dramatically different!

Remarkably, in experiments involving a source of quantum particles, Bell's inequality (8) can be violated. This means that there exist quantum experiments, the statistics of which cannot be written in the form (6). This is quantum nonlocality, a phenomenon repeatedly observed experimentally, which has many applications in quantum information processing. 
A crucial feature of quantum correlations is that they satisfy the no-signalling principle, represented by conditions of the form (3). This is indeed fundamental, as it ensures that quantum mechanics is compatible with relativity. It turns out, however, that there exist correlations which are stronger than those allowed in quantum mechanics, which nevertheless satisfy the nosignalling principle. Such correlations, discovered by Popescu and Rohrlich ${ }^{6}$, are often referred to as super-quantum correlations or nonlocal boxes.

For Bayesian games, the possibility of having access to nonlocal correlations, for instance using entanglement, has important implications. First let us imagine that the players can share quantum advice, that is, the advisor is able to produce entangled particles and to send them to the players, who then perform local measurements on their particles. As the statistics of such measurements can in general not be reproduced by any classical local model, the players now have access to strategies that would be impossible in the case of a classical advisor. Thus, players sharing quantum advice can outperform any classical players. More formally, this means that the space of payoff functions for players sharing quantum advice can become larger than the space of payoff functions for classical players. In case the average payoff function of one (or more) player corresponds to a Bell inequality, then quantum resources give an advantage to the players. Interestingly, however, even in the case none of the payoff functions corresponds to a Bell inequality (i.e., the highest possible payoff can be reached classically), it is still possible in certain cases to obtain a quantum advantage.

Going beyond quantum mechanics, it is relevant to consider general nonlocal resources in the context of Bayesian games. In general, this allows for novel strategies, which can outperform both classical and quantum strategies. Hence the space of payoffs achievable with no-signalling strategies is in general larger than in the case of quantum strategies.

Finally, allowing for quantum or super-quantum strategies also provides novel correlated equilibrium points to the game. Such points are referred to as quantum Nash equilibria and nosignalling Nash equilibria.

Below we will illustrate these ideas by discussing a few simple examples of Bayesian games featuring a quantum advantage and a no-signalling advantage.

Game where payoff functions are Bell inequalities. We first consider a simple game between two players, characterized as follows. For each player there are only two possible types, $X_{1}=0,1$ for the player 1 (from now called Alice), and $X_{2}=0,1$ for the player 2 (from now called Bob). The set of possible actions is also composed of two elements only: actions $A_{1}=0,1$ for Alice, and $A_{2}=0,1$ for Bob. There are thus four possible states of Nature, and we will consider them equally likely: $\mu\left(X_{1}, X_{2}\right)=1 / 4$ for $X_{1}, X_{2}=0,1$.

Next we define the payoff function of Alice to be given by

$$
f_{1}\left(X_{1}, X_{2}, A_{1}, A_{2}\right)=\left\{\begin{array}{cc}
+4 & \text { if } A_{1} \oplus A_{2}=X_{1} X_{2} \\
-4 & \text { otherwise }
\end{array}\right.
$$

where $\oplus$ designates addition modulo 2 . Thus, the average payoff function of Alice is given by

$$
\begin{gathered}
F_{1}=E\left(X_{1}=X_{2}=0\right)+E\left(X_{1}=0, X_{2}=1\right) \\
+E\left(X_{1}=1, X_{2}=0\right)-E\left(X_{1}=X_{2}=1\right)
\end{gathered}
$$

where we have defined the correlation function

$$
E\left(X_{1}, X_{2}\right)=P\left(A_{1}=A_{2} \mid X_{1}, X_{2}\right)-P\left(A_{1} \neq A_{2} \mid X_{1}, X_{2}\right)
$$

We will consider the game to be symmetric, hence the payoff function of Bob is the same as that of Alice, i.e., $F_{1}=F_{2}$.
Now it turns out that the function (11) is very well known in quantum mechanics. It is the basis of the simplest Bell inequality, derived in 1969 by Clauser-Horne-Shimony-Holt $(\mathrm{CHSH})^{26}$. The $\mathrm{CHSH}$ Bell inequality reads $F_{1} \leq 2$. In quantum mechanics, by performing judicious measurements on a singlet state, of the form $\left|\psi_{-}\right\rangle=\left(|0\rangle_{A}|1\rangle_{B}-|1\rangle_{A}|0\rangle_{B}\right) / \sqrt{2}$, it is possible to obtain the following set of correlation functions:

$$
E_{\psi_{-}}\left(X_{1}, X_{2}\right)=(-1)^{X_{1} X_{2}} \frac{1}{\sqrt{2}}
$$

This leads to $F_{1}=2 \sqrt{2}>2$, hence violating the $\mathrm{CHSH}$ inequality. Therefore, the space of possible payoffs in the case of quantum advice is clearly larger than in the classical case, as there exist individual values of the payoff function which are not attainable classically.

Moreover, the quantum setting provides here a new equilibrium point. Indeed, it turns out that the value of $F_{1}=F_{2}=2 \sqrt{2}$ is in fact the maximum that is achievable in quantum mechanics ${ }^{27}$. Therefore, the point $F_{1}=F_{2}=2 \sqrt{2}$ represents a quantum correlated equilibrium point, as it is impossible for Alice or Bob to obtain a larger payoff by adopting any other strategy.

Next, let us consider super-quantum correlations. It turns out that such correlations can give rise to maximal violation of the $\mathrm{CHSH}$ inequality, reaching $\mathrm{CHSH}=4$. Thus, players sharing such super-quantum correlations can outperform quantum players in the above game, and reach $F_{1}=F_{2}=4$, achieving the highest possible average payoff. In particular, this is achieved using no-signalling correlations known as the Popescu-Rohrlich (PR) box, characterized by $E_{\mathrm{PR}}\left(X_{1}, X_{2}\right)=(-1)^{X_{1} X_{2}}$. Indeed, the point $F_{1}=F_{2}=4$ is a no-signalling Nash equilibrium, as no higher payoffs are possible.

Game where none of the payoff functions is a Bell inequality. Let us now consider an asymmetric variation of the above game. We take again $\mu\left(X_{1}, X_{2}\right)=1 / 4$ for $X_{1}, X_{2}=0,1$. The payoff functions for Alice and Bob will now be different:

$$
\begin{gathered}
f_{1}\left(X_{1}, X_{2}, A_{1}, A_{2}\right)=\left\{\begin{array}{cc}
4\left(1-X_{1}\right) & \text { if } A_{1} \oplus A_{2}=X_{1} X_{2} \\
-4\left(1-X_{1}\right) & \text { otherwise }
\end{array}\right. \\
f_{2}\left(X_{1}, X_{2}, A_{1}, A_{2}\right)=\left\{\begin{array}{cc}
+4 X_{1} & \text { if } A_{1} \oplus A_{2}=X_{1} X_{2} \\
-4 X_{1} & \text { otherwise }
\end{array}\right.
\end{gathered}
$$

Hence, we obtain the following average payoffs

$$
\begin{aligned}
& F_{1}=E\left(X_{1}=X_{2}=0\right)+E\left(X_{1}=0, X_{2}=1\right) \\
& F_{2}=E\left(X_{1}=1, X_{2}=0\right)-E\left(X_{1}=X_{2}=1\right)
\end{aligned}
$$

It is straightforward to see that $F_{1} \leq 2$ and $F_{2} \leq 2$ for any possible strategy (classical and quantum). However, in the case of classical advice, it holds that $F_{1}+F_{2} \leq 2$, which is simply the $\mathrm{CHSH}$ Bell inequality. Note that this inequality is a facet of the space of payoffs. Using quantum advice, in particular the optimal CHSH strategy given in (12), one has that $F_{1}=F_{2}=\sqrt{2}<2$, but $F_{1}+F_{2}=2 \sqrt{2}>2$. Thus, we obtain a set of average payoffs which cannot be obtained classically, although each payoff is individually compatible with a classical model (see Fig. 2).

Note that the space of payoff functions in the case of quantum advice is not a polytope in general. Here it can be checked that all points satisfying $F_{1}^{2}+F_{2}^{2}=4$ can be attained by performing judicious measurements on a singlet state.

Finally, considering advice based on super-quantum correlations leads to even better strategies. Again, the PR box allows both players to reach the optimal payoff, i.e., achieving $F_{1}=F_{2}=2$. This point is a no-signalling Nash equilibrium.

A more realistic example. In our final example, the players are two companies, both interested in buying jointly some pieces of land, potentially rich in a certain resource. Company A has 


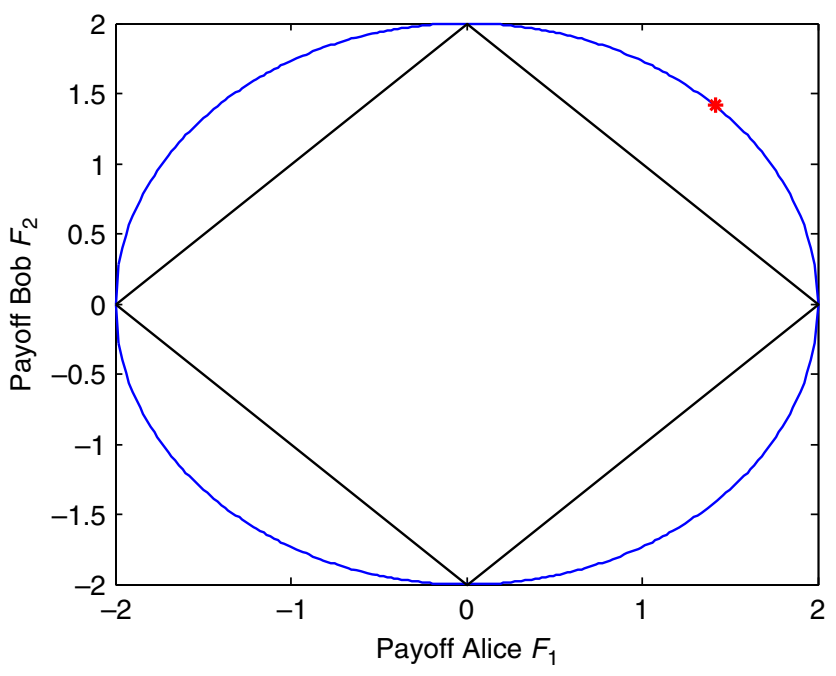

Figure 2 | Space of possible average payoffs functions. The pairs of payoffs achievable with classical advice are represented by the back polytope (square). Players sharing quantum advice have access to a strictly larger set of possible payoff function (blue circle), and hence have an advantage over classical players. The red star represents the particular quantum strategy mentioned in the main text. Players having access to super-quantum advice can reach all points, in particular the point $F_{1}=F_{2}=2$, a no-signalling Nash equilibrium.

Table 1 | Payoffs for the more realistic example.

\begin{tabular}{|c|c|c|c|c|}
\hline & \multicolumn{2}{|c|}{ Low supply } & \multicolumn{2}{|c|}{ High supply } \\
\hline & No bid & Bid & No bid & Bid \\
\hline \multicolumn{5}{|l|}{ Low cost } \\
\hline No bid & $(0,0)$ & $(4,4)$ & $(0,0)$ & $(2,2)$ \\
\hline Bid & $(4,4)$ & $(2,2)$ & $(2,2)$ & $(1,1)$ \\
\hline \multicolumn{5}{|l|}{ High cost } \\
\hline No bid & $(0,0)$ & $(2,2)$ & $(0,0)$ & $(-2,-2)$ \\
\hline Bid & $(2,2)$ & $(1,1)$ & $(-2,-2)$ & $(-2,-2)$ \\
\hline \multicolumn{5}{|c|}{$\begin{array}{l}\text { Two companies are interested in buying jointly some pieces of land, potentially rich in some } \\
\text { resource. Company A (vertically) will extract the resource, and the production cost can be low } \\
\text { or high. Company B (horizontally) will sell the product, and the supply on the market can be low } \\
\text { or high. During the sale, both companies must bid simultaneously. A piece of land is sold to } \\
\text { them when at least one of the companies places a bid. The table gives all pairs of payoffs, }\left(f_{A}, f_{B}\right) \\
\text { for all possible types and strategies of the players. }\end{array}$} \\
\hline
\end{tabular}

expertise in extracting this resource, while company $\mathrm{B}$ has expertise in selling and distributing it. Hence an association is potentially profitable for both companies, which would then share the net profit equally.

For company A, the price of production may vary, depending on various parameters, which represent the type of company A. Here the extraction cost can be either low or high (with equal probability), which an expert of company A can evaluate. For company $\mathrm{B}$, the type is the supply on the market, which can be either low or high (with equal probability), evaluated by an expert of company B. Note that the type of each company is private, as each company is reluctant to let the other know about how much benefit it could potentially make.

The sale is organized as follows. Both companies will be asked simultaneously, to bid or not on a particular piece of land. If at least one company bids, the piece of land is sold. All money that is bid is retained, hence if both companies bid, their profit is lower than if only one of them bids. If both the extraction cost and supply on the market are low, the profit will be high. If the extraction cost is low but the supply is high (or conversely) the profit is medium. If both the extraction cost and supply are high, the companies go bankrupt if they bid. The payoff functions for this Bayesian game are given in Table 1.

It is not difficult to see that, in the case the companies have access to classical advice, the largest possible average payoff for each company is $F_{A, B}=3 / 2$. However, having access to quantum advice, the companies can achieve $F_{A, B} \simeq 1.5365>3 / 2$. This is achieved by performing suitably chosen local measurements on a singlet state. Moreover, this point represents a quantum correlated Nash equilibrium, as no better score can be achieved.

\section{Discussion}

We have discussed a strong connection between Bell nonlocality and Bayesian games, showing that similar concepts appear in both fields. This led us to characterize games for which players sharing advice based on nonlocal correlations, for instance using quantum entanglement, can outperform players sharing (any possible) classical advice. Considering the quantum case, it is important to emphasize that the advantage provided by quantum resources is here fully general. Hence it does not rely on any specific restrictions, contrary to previous approaches to quantum games ${ }^{18}$, which then lead to controversy ${ }^{19-22}$. The main point is that these approaches focused on games with complete information (such as Prisoner's dilemma), where the notion of type is not present, in contrast to Bayesian games. This is perhaps expressed even more clearly from the point of view of nonlocality: a Bell test can separate quantum from classical predictions only if each observer can choose between several possible measurements to perform.

Finally, we believe that the connection presented here may also benefit nonlocality. Besides providing new potential applications for quantum nonlocality, along with quantum communications $s^{3,4}$ and communication complexity ${ }^{5}$, it also raises interesting issues, in particular the possibility of detecting nonlocal correlations via a set of Bell type inequalities, rather than from a single Bell parameter.

\section{References}

1. Bell, J. S. Speakable and Unspeakable in Quantum Mechanics (Cambridge University Press, 1987).

2. Aspect, A. Bell's inequality test: more ideal than ever. Nature 398, 189-190 (1999).

3. Ekert, A. K. Quantum cryptography based on Bell's theorem. Phys. Rev. Lett. 67, 661-663 (1991).

4. Acin, A. et al. Device-independent security of quantum cryptography against collective attacks. Phys. Rev. Lett. 98, 230501 (2007).

5. Buhrman, H., Cleve, R., Massar, S. \& de Wolf, R. Nonlocality and communication complexity. Rev. Mod. Phys. 82, 665-698 (2010).

6. Popescu, S. \& Rohrlich, D. Quantum nonlocality as an axiom. Found. Phys. 24, 379-385 (1994).

7. Barrett, J. et al. Nonlocal correlations as an information-theoretic resource. Phys. Rev. A 71, 022101 (2005).

8. Popescu, S. Quantum mechanics: Why isn't nature more non-local? Nat. Phys. 2, 507-508 (2006).

9. Pawlowski, M. et al. Information causality as a physical principle. Nature 461, 1101-1104 (2009).

10. Harsanyi, J. C. Games with incomplete information played by Bayesian players Manage. Sci. 14, 159-183 (Part I): 14 (5) 320-334 (Part II), 14 (7): 486-502 (Part III), (1967).

11. Cheon, T. \& Iqbal, A. Bayesian Nash equilibria and Bell inequalities. J. Phys. Soc. Jpn 77, 024801 (2008).

12. Iqbal, A., Cheon, T. \& Abbott, D. Probabilistic analysis of three-player symmetric quantum games played using the Einstein-Podolsky-Rosen-Bohm setting. Phys. Lett. A 372, 6564-6577 (2008).

13. Flitney, A. P. et al. Equivalence between Bell inequalities and quantum Minority game. Phys. Lett. A 373, 521-524 (2009).

14. Iqbal, A. \& Abbott, D. Constructing quantum games from a system of Bell's inequalities. Phys. Lett. A 374, 3155-3163 (2010).

15. Hill, C. D., Flitney, A. P. \& Menicucci, N. C. A competitive game whose maximal Nash-equilibrium payoff requires quantum resources for its achievement. Phys. Lett. A 374, 3619-3624 (2010).

16. Schmid, C. et al. Experimental implementation of a four-player quantum game. New J. Phys. 12, 063031 (2010).

17. Chappell, J. M., Iqbal, A. \& Abbott, D. Analyzing three-player quantum games in an EPR type setup. PLoS ONE 7, e36404 (2012). 
18. Eisert, J., Wilkens, M. \& Lewenstein, M. Quantum Games and Quantum Strategies. Phy. Rev. Lett. 83, 3077-3080 (1999).

19. Benjamin, S. C. \& Hayden, P. M. Comment on Quantum Games and Quantum Strategies. Phys. Rev. Lett. 87, 069801 (2001).

20. Eisert, J., Wilkens, M. \& Lewenstein, M. Reply. Phys. Rev. Lett. 87, 069802 (2001).

21. van Enk, S. J. \& Pike, R. Classical rules in quantum games. Phys. Rev. A 66, 024306 (2002).

22. Aharon, N. \& Vaidman, L. Quantum advantages in classically defined tasks. Phys. Rev. A 77, 052310 (2008).

23. Osborne, M. J. An Introduction to Game Theory (Oxford University Press, 2002).

24 . Forges, F. Correlated equilibrium in games with incomplete information revisited. Theory Decis. 61, 329-344 (2006).

25. Aumann, R. Subjectivity and correlation in randomized strategies. J. Math Econ. 1, 67-96 (1974).

26. Clauser, J. F., Horne, M. A., Shimony, A. \& Holt, R. Proposed experiment to test local hidden-variable theories. Phys. Rev. Lett. 23, 880-884 (1969).

27. Tsirelson, B. S. Quantum generalizations of Bell's inequality. Lett. Math. Phys. 4, 93-100 (1980).

\section{Acknowledgements}

We are very grateful for illuminating discussions with David Leslie and John McNamara. We acknowledge financial support from the UK EPSRC, the EU DIQIP, the Swiss National Science Foundation (grant PP00P2_138917) and the Templeton Foundation.

\section{Author contributions}

N.B. and N.L. performed the theoretical analysis and wrote the paper.

\section{Additional information}

Competing financial interests: The authors declare no competing financial interests.

Reprints and permission information is available online at http://npg.nature.com/ reprintsandpermissions/

How to cite this article: Brunner, N. and Linden, N. Connection between Bell nonlocality and Bayesian game theory. Nat. Commun. 4:2057 doi: 10.1038/ncomms3057 (2013). 